\title{
Improving Wireless Packet Throughput Using Hybrid-II ARQ and Physical Layer Side Information
}

\author{
Cheing-hong Lin and Jingshown Wu \\ Room 519 \\ Department of Electrical Engineering and \\ Graduate Institute of Communication Engineering \\ National Taiwan University \\ Taipei, Taiwan 10617 \\ R.O.C
}

\begin{abstract}
In this paper, the physical layer side information is employed to improve the throughput of the hybrid-II ARQ protocol in the binary phase shift keying wireless communication system. With the combination of the hybrid-II ARQ and the n-bit-marked error correction scheme, the packet throughput is improved significantly. The numerical examples show that the throughput of this combination scheme may have a maximum gain about 0.26 .
\end{abstract}

\section{INTRODUTION}

Wireless communications possess the convenient mobility property, which is very desirable. However, the air link, which may be effected by noise, multi-path fading, Doppler frequency shift, introduces significant errors in a mobile computing system. The conventional automatic-repeat-request (ARQ) protocol, which is designed for the wired transmission environment, can effectively control errors for the higher layers. Because of the high error rate for air link, the ARQ protocol becomes not very effective as it used in the wired transmission environment [1]. The forward-error-correction (FEC) scheme is a well-known method to control errors. Directly applying FEC scheme and the ARQ protocol on a mobile computing system can reduce errors at the expenses of transmission bandwidth, because a packet with FEC has a lot of redundant bits [2]. When the bit error rate (BER) is not so high, say $10^{-4}$, this direct combination of ARQ and FEC wastes the bandwidth, which is very undesirable.

The Hybrid-II ARQ scheme using FEC codes, which is devised based on the concept that the parity-check bits for error correction are sent to the receiver only when needed, increases the bandwidth efficiency and reduces the average packet delay [3]. Two linear codes are used in this scheme; one is a high-rate $(\mathrm{B}, \mathrm{B}-\mathrm{k})$ code $\mathrm{C}_{0}$, which is designed for error detection only, the other is an invertible half-rate $(2 \mathrm{k}, \mathrm{k})$ code $\mathrm{C}_{1}$, which is designed for simultaneous error correction and error detection. The packet formats are depicted in Fig.1.

The operation procedure of the hybrid-II ARQ is described as following. At first, the transmitting node sends the packet with the high-rate code. When the packet is received successfully, the receiving node sends an acknowledgement (ACK) back to the transmitting node. Otherwise, the receiving node sends a negative acknowledgement (NAK) back and the transmitting node will transmit the corresponding invertible half-rate code for correction. If the packet is successfully corrected, the receiving node sends an ACK back. On the contrary, the receiving node sends a NAK for the retransmission of the high-rate code packet and drops the former one. If the retransmitted one is correct, an ACK will be sent. Otherwise, it will be corrected by the former corresponding invertible half-rate code. If the packet still can't be corrected, the packet with the corresponding invertible

'The work is supported in part by the National Science Council, R.O.C under the grant NSC 88-2213-E-002-081

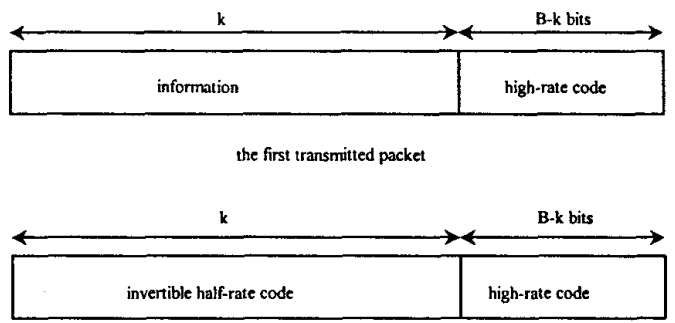

the second transmitted packet

Fig.1. The packet formats of hybrid-II ARQ.

half-rate code will be dropped and a NAK will be sent for the retransmission of the packet with the corresponding invertible half-rate code. This procedure will be repeated until the packet received successfully [4].

On the other hand, the n-bit-marked error correction scheme employs the physical layer side information to increase the correctness of every transmitted packet for improving the throughput [5]. In this scheme, the transmitting node doesn't have to change the structures of the physical layer and the data link layer. In the receiving node, a side information generator is added. The receiver structure with binary phase shift keying demodulator and the side information generator is shown in Fig.2. We mark the bit, which is in Conditions II and III.

In this paper, we combine the n-bit-marked error correction and the hybrid-II ARQ scheme. The operation procedure of this combined scheme is very similar to that of the hybrid-II ARQ scheme. The only difference is that we use the n-bit-marked error correction scheme to increase the correctness of every hybrid-II ARQ packet to improve the throughput. The operation procedure of this combined scheme is described as following. At first, the transmitting node sends the packet with the high-rate code. If the packet is received successfully by employing the n-bit-marked error correction scheme, the receiving node sends an ACK back to the transmitting node. Otherwise, it sends a NAK back to request for transmitting the packet with the corresponding invertible half-rate code. This packet is also received by employing the n-bit-marked error correction scheme. If it is correct or can be corrected by the n-bit-marked error correction scheme, the receiving node sends an ACK back. If not, it also can be used to correct the information packet. If the packet is successfully corrected by the corresponding invertible half-rate code, the receiving node sends an ACK back. On the contrary, the receiving node sends a NAK for the retransmission of the packet with high-rate code and drops the former one. If the retransmitted one is correct or can be corrected by the n-bit-marked error correction scheme, an ACK will be sent. 


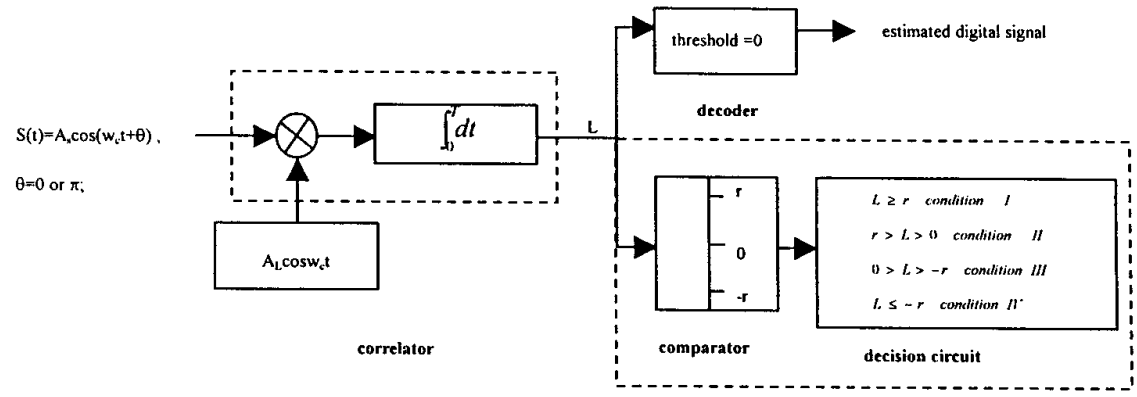

side information generator

Fig.2: receiver structure

Otherwise, the former packet with the corresponding invertible half-rate code will be used to correct the packet.. If the packet still can't be corrected, the packet with the corresponding invertible half-rate code will be dropped and a NAK will be sent for the retransmission of the packet with the corresponding invertible half-rate code. This procedure will be repeated until the packet received successfully.

\section{THE THROUGHPUT ANALYSIS}

The throughput of the hybrid-II ARQ scheme is effected by the values of $q_{I}$ and $q_{2}$. $q_{I}$ is the conditional probability that the packet can be recovered by the second transmitted packet when the first transmitted packet is detected error. $q_{2}$ is the conditional probability that the packet can be recovered by the third transmitted packet when the first and second transmitted packets are detected error and can not be recovered by the packet with the invertible half-rate code. For the combination the hybrid-II ARQ and the n-bit-marked scheme, $q_{l}$ can be expressed as

$$
q_{1}=\left(p_{c}+p_{n}\right)+p_{d d c 1} /\left(1-p_{c}-p_{n}\right)
$$

where $P_{c}$ is the probability of the first packet is received successfully without invoking the correction algorithm. $P_{n}$ is the probability that the erroneous packet is successfully recovered by the $\mathrm{n}$-bit-marked error correction scheme. $P_{\text {cddcl }}$ is the probability that the first and second transmitted packets are erroneous and can't be corrected by the n-bit-marked error correction scheme but can be recovered by invertible high-rate code. $P_{d d c /}$ can be expressed as

$$
p_{d d d}=p_{d d c}-p_{A}-p_{A 1}+p_{B}
$$

where $P_{d d c}$ is the probability that the first and second transmitted packets are erroneous but can be recovered by invertible high-rate code. $P_{A}$ is the probability that the first and second transmitted packets are detected errors, the first transmitted packet can be recovered by the n-bit-marked error correction scheme and the first and second packets can be recovered by the half-rate code. $P_{A I}$ is the probability that the first and second transmitted packets are detected error, the second transmitted packet can be recovered by the n-bit-marked error correction scheme and the first and second packets can be recovered by the half-rate code. $P_{B}$ is the probability that the first and second transmitted packets are detected error, both can be recovered by the n-bit-marked error correction scheme, and the first packet can be recovered by the half-rate code. The probabilities, $P_{A}$ and, $P_{A l}$ are equal. $P_{d d c^{\prime} l}$ can also be expressed as

$$
p_{d d c 1}=p_{d d c}-2 p_{A}+p_{B}
$$

We assume that there are $N$ marked bits in the first transmitted packet and among them $h$ marked bits are in the first $k$ bits of the packet. Let $t$ be the correction power of the invertible half-rate code and $n$ is the maximum number of marked bits of the n-bit-marked error correction scheme. The probability $P_{A}$ is given by

$$
\begin{aligned}
& p_{A}=\sum_{h=0}^{n} \sum_{N=h}^{n} C_{h}^{k} C_{N-h}^{B-k} p_{1}^{B-N} \bullet \\
&\left\{\begin{array}{c}
\sum_{j=0}^{h} C_{j}^{h} p_{2}^{h-j} p_{3}^{j}\left(p_{2}+p_{3}\right)^{N-h}\left[\sum_{i=0}^{1-j} C_{i}^{k} p_{e}^{i}\left(1-p_{e}\right)^{k-i}-\left(1-p_{e}\right)^{B}\right] \\
-p_{2}^{N}\left[\sum_{i=0}^{1} C_{i}^{k} p_{e}^{i}\left(1-p_{e}\right)^{k-i}-\left(1-p_{e}\right)^{B}\right]
\end{array}\right\} ; j \leq t,
\end{aligned}
$$

where $P_{e}$ is the BER, $B$ is the number of bit in a packet. $P_{l}$ is the probability that the signal energy of a received bit is larger than $\mathrm{r}$ (the threshold of side information generator). $P_{2}$ is the probability that the energy of a received bit is fallen in the $(0, r)$ region. $P_{3}$ is the probability that a received bit is fallen in the $(0$, -r) region. The detailed derivation of $P_{A}$ is presented in the Appendix.

$P_{B}$, whose derivation is similar to that of $P_{A}$, is given by

$$
\begin{aligned}
p_{B}= & \sum_{h=0}^{n} \sum_{N=h}^{N} C_{h}^{k} C_{N-h}^{R-k} p_{1}^{B-N} \bullet \\
& \left\{\sum_{j=0}^{h} C_{j}^{h} p_{2}^{h-j} p_{3}^{j}\left(p_{2}+p_{3}\right)^{N-h} p_{R 1}-p_{2}^{N} p_{R 2}\right\} ; j \leq t,
\end{aligned}
$$




$$
\begin{aligned}
p_{k 1}= & \sum_{N_{1}=1}^{n} \sum_{h_{1}=0}^{N_{1}} C_{h_{1}}^{k} C_{N_{1}-h_{1}}^{B-k} p_{1}{ }^{B-N_{1}} \bullet \\
& \left\{\sum_{i=0}^{h_{1}} C_{1}^{h_{1}} p_{2}^{h_{1}-i} p_{3}{ }^{\prime}\left(p_{2}+p_{3}\right)^{N_{1}-h_{1}}-p_{2}{ }^{N_{1}}\right\} ; i \leq t-j, \\
p_{R 2}= & \sum_{N_{1}=1}^{n} \sum_{h_{1}=0}^{N_{1}} C_{h_{1}}^{k} C_{N_{1}-h_{1}}^{B-k} p_{1}^{B-N_{1}} \bullet \\
& \left\{\sum_{i=1}^{h_{1}} C_{i}^{h_{1}} p_{2}^{h_{1}-i} p_{3}{ }^{i}\left(p_{2}+p_{3}\right)^{N_{1}-h_{1}}-p_{2}{ }^{N_{1}}\right\} ; i \leq t,
\end{aligned}
$$

where $P_{R I}$ is the probability that a packet with one to $(t-j)$ errors in the first $k$ bits can be recovered by the n-bit-marked error correction scheme and $P_{R 2}$ is the probability that a packet with one to $\mathrm{t}$ errors in the first $\mathrm{k}$ bits can be recovered by the n-bit-marked error correction scheme. Similarly, when the n-bit-marked error correction scheme is employed, $q_{2}$ is given by

$$
\begin{aligned}
q_{2}= & \left(p_{c}+p_{n}\right)+\frac{1}{\left(1-p_{c}-p_{n}\right)\left(1-q_{1}\right)} \\
& \cdot \sum_{i=0}^{i-1} \Delta_{1}(i) s_{1}(t-i)\left[1-\Delta(0)-s(t-i)-s_{2}(i)\right]
\end{aligned}
$$

and

$$
\begin{gathered}
\Delta(i)=C_{i}^{k} p_{e}^{i}\left(1-p_{e}\right)^{k-i} \\
s(j)=\sum_{i=1}^{j} \Delta(l) \\
\Delta_{1}(i)=\Delta(i)-\sum_{N=i}^{n} \sum_{h=i}^{N} C_{h}^{k} C_{i}^{h} C_{N-h}^{B-k} p_{1}{ }^{B-N} p_{2}{ }^{h-i} p_{3}{ }^{i}\left(p_{2}+p_{3}\right)^{N-h} \\
s_{1}(j)=\sum_{i=1}^{K} \Delta_{1}(l) \\
s_{2}(j)=\sum_{l=(t-j+1)}^{n} \sum_{N=l}^{n} \sum_{h=l}^{N} C_{h}^{k} C_{i}^{h} C_{N-h}^{B-k} p_{1}{ }^{B-N} p_{2}{ }^{h-i} p_{3}{ }^{\prime}\left(p_{2}+p_{3}\right)^{N-h}
\end{gathered}
$$

where $P_{c}$ is the probability of the first packet is received successfully without invoking the correction algorithm. $P_{n}$ is the probability that the erroneous packet is successfully recovered by the n-bit-marked error correction scheme. $\Delta$ (i) is the event that the first $k$ bits of the third transmitted packet contain exactly $i$ errors. $\Delta,(i)$ is the event that the first $k$ bits of the third transmitted packet contain exactly $i$ errors and can not be corrected by the n-bit-marked error correction scheme. $S(j)$ is the event that the first $\mathrm{k}$ bits of the third transmitted packet contain one to i errors. $S_{(}(j)$ is the event that the first k bits of the third transmitted packet contain one to $i$ errors and can not be corrected by the n-bit-marked error correction scheme. $S_{2}(\mathrm{j})$ is the event that the first $\mathrm{k}$ bits of the third transmitted packet contain $(t-j+l)$ to $\mathrm{k}$ errors and can be corrected by the n-bit-marked error correction scheme.

Denote that

$$
\begin{aligned}
& \alpha=I-\left(I-p_{c}-p_{n}\right)\left(I-q_{1}\right) \\
& \beta=I-\left(I-p_{c}-p_{n}\right)\left(I-q_{I}\right)\left(I-q_{2}\right) \\
& \gamma=q_{I}+q_{2}-q_{I} q_{2}
\end{aligned}
$$

Then the throughput of the combination scheme with receiver buffer of size $N$ has a lower bound as

$$
\eta \geq \delta_{0} /\left(\delta_{0}+\delta_{1}+\delta_{2} N\right)
$$

where

$$
\begin{aligned}
& \delta_{0}=\gamma\left(1-\gamma \beta^{N-1}\right) /(1-\beta) \\
& \delta_{I}=\left(p_{c}+p_{n}\right) q_{l} \alpha^{N-2}+\left(p_{c}+p_{n}\right)\left(1-q_{l}\right) q_{2} \beta^{N-2} \\
& \delta_{2}=2-\left(p_{c}+p_{n}\right) q_{l} \alpha^{N-2}-\alpha \gamma \beta^{N-2}
\end{aligned}
$$

The throughput versus BER with $t=3,5$ or 10 are plotted in Figs.3 5. In Fig.3, the maximum gain is 0.26 . Here the gain is defined as the difference of the throughput of the hybrid-II ARQ and that of the combination of the hybrid-II ARQ and the n-bit-marked error correction scheme. The maximum gains are 0.24 and 0.17 for $t=5, n=10$ and $t=10, n=15$ as shown in Figs. 4 and 5. It is observed that the proposed scheme can increase the packet throughput significantly at BER around $1.5 \times 10^{-3}$, where the throughput of the conventional selective repeat ARQ is almost zero.

\section{CONCLUSION}

In this paper, we combine the hybrid-II ARQ and the n-bit-marked error correction scheme to improve the throughput performance. We take the advantages of using side information and FEC codes. The numerical results show that this scheme can improve the throughput significantly. The maximum gain of this combination scheme is 0.26 at BER around $1.5 \times 10^{-3}$. This scheme needs computation power in the receiver. It is expected that when the cost effective digital signal processing integrated circuit chip is available, this scheme can be practical.

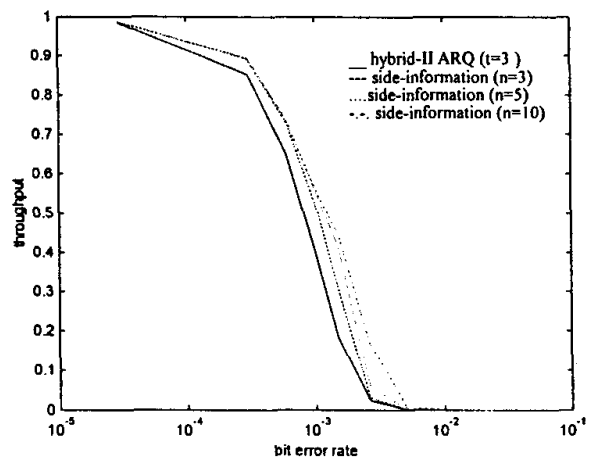

Fig.3.The throughput of the combination of $n$-bit-marked error correction ( $n=3,5$ or 10$)$ and hybrid-II ARQ with $t=3$. The buffer size is 128 and the packet size is 524 . 


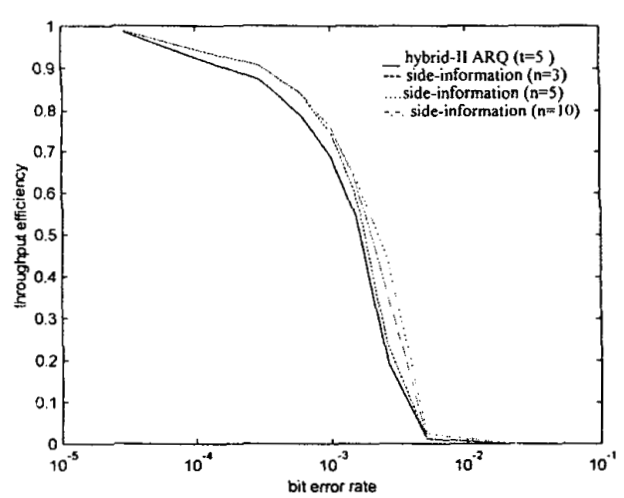

Fig.4.The throughput of the combination of $n$-bit-marked error correction ( $\mathrm{n}=3,5$ or 10$)$ and hybrid-II ARQ with $\mathrm{t}=5$. The buffer size is 128 and the packet size is 524 .

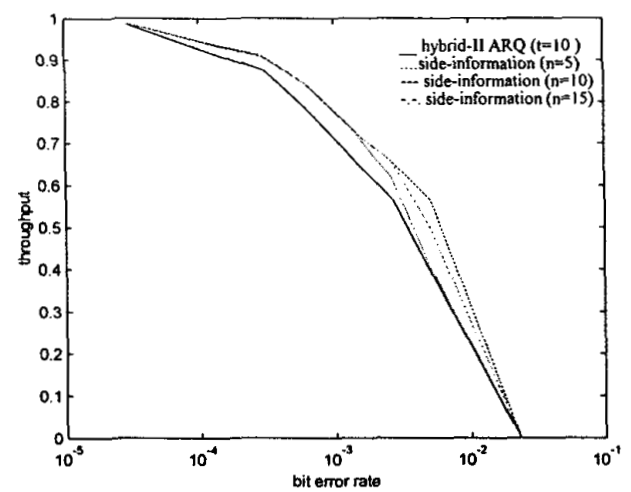

Fig.5.The throughput of the combination of n-bit-marked error correction ( $\mathrm{n}=5,10$ or 15$)$ and hybrid-II ARQ with $t=10$. The buffer size is 128 and the packet size is 524 .

\section{APPENDIX}

First, we assume that $\mathrm{N}=1$ and $\mathrm{h}=0$. Then $P_{A}$ can be expressed as

$$
\begin{aligned}
P_{A} & =\left[P_{1}^{k} C_{l}^{B-k} P_{I}^{B-k-1} P_{3}\right\}\left\{\left[\sum_{i=0}^{1} C_{i}^{k} P_{e}^{i}\left(1-P_{e}\right)^{k-i}\right]-\left(1-P_{e}\right)^{B}\right\} \\
& =\left[C_{l}^{B-k} P_{l}^{B-1} P_{3}\right]\left\{\left[\sum_{i=0}^{f} C_{i}^{k} P_{e}^{i}\left(1-P_{e}\right)^{k-i}\right]-\left(1-P_{e}\right)^{B}\right\}
\end{aligned}
$$

If $\mathrm{N}=2$ and $\mathrm{h}=0$, then $P_{A}$ can be expressed as

$$
\begin{aligned}
P_{A} & =\left[P_{1}^{k} C_{2}^{B-k} P_{1}^{B-k-2}\left(2 P_{2} P_{3}+P_{3}{ }^{2}\right)\right\}\left[\left[\sum_{i=0}^{1} C_{t}^{k} P_{e}^{\prime}\left(1-P_{e}\right)^{k-1}\right]-\left(1-P_{*}\right)^{B}\right\} \\
& =\left\{C_{2}^{B-k} P_{1}^{B-2}\left(P_{2}+P_{3}\right)^{2}-P_{2}{ }^{2}\right]\left\{\left[\sum_{i=0}^{1} C_{i}^{k} P_{e}^{\prime}\left(1-P_{e}\right)^{k-1}\right]-\left(1-P_{e}\right)^{B}\right\}
\end{aligned}
$$

If $\mathrm{N}=\mathrm{n}$ and $\mathrm{h}=0$, then $P_{A}$ can be expressed as

$$
P_{A}=\left\{C_{n}^{B-k} P_{1}^{B-n}\left[\left(P_{2}+P_{3}\right)^{n}-P_{2}^{n}\right]\left[\left[\sum_{i=0}^{1} C_{i}^{k} P_{e}^{i}\left(I-P_{e}\right)^{k-1}\right]-\left(1-P_{e}\right)^{B}\right\}\right.
$$

Therefore, when $\mathrm{h}=0$, the total probability of $P_{A}$ can be expressed as

$P_{A}=\sum_{N=1}^{n}\left\{C_{N}^{B-k} P_{1}^{B-N}\left[\left(P_{2}+P_{3}\right)^{N}-P_{2}^{N}\right):\left[\left[\sum_{i=0}^{1} C_{i}^{k} P_{e}^{i}\left(I-P_{e}\right)^{k-i}\right]-\left(I-P_{e}\right)^{B}\right\}\right.$

Next, we assume $\mathrm{N}=1$ and $\mathrm{h}=1$ and $P_{A}$ is expressed as

$$
P_{A}=\left[C_{1}^{k} P_{T}^{B \sim 1} P_{3}\right]\left\{\left[\left\{\sum_{i=0}^{t-1} C_{i}^{k} P_{e}^{i}\left(1-P_{e}\right)^{k-i}\right]-\left(1-P_{e}\right)^{B}\right\}\right.
$$

If $\mathrm{N}=2$ and $\mathrm{h}=1$, then $P_{A}$ is given by

$$
\begin{aligned}
& P_{A}=\left[C_{I}^{k} P_{I}^{k-1} P_{3} C_{I}^{B-k} P_{l}^{B-k-1}\left(P_{2}+P_{3}\right)\right]\left\{\left[\sum_{i=0}^{1-I} C_{i}^{k} P_{e}^{i}\left(I-P_{e}\right)^{k-i}\right]-\left(I-P_{e}\right)^{B}\right\} \\
& +\left[C_{I}^{k} P_{I}^{k-1} P_{2} C_{I}^{B-k} P_{I}^{B-k-1} P_{3}\right\}\left\{\left[\sum_{i=0}^{\prime} C_{i}^{k} P_{e}^{i}\left(1-P_{e}\right)^{k-i}\right]-\left(I-P_{e}\right)^{B}\right\} \\
& =\left[C_{l}^{k} C_{l}^{B-k} P_{1}^{B-2} P_{3}\left(P_{2}+P_{3}\right)\right]\left[\left[\sum_{i=0}^{t-1} C_{i}^{k} P_{e}^{i}\left(1-P_{e}\right)^{k-i}\right]-\left(1-P_{e}\right)^{B}\right\} \\
& +\left[C_{l}^{k} C_{1}^{B-k} P_{I}^{B-2} P_{2} P_{3}\right]\left\{\left[\sum_{i=0}^{1} C_{i}^{k} P_{e}^{i}\left(I-P_{e}\right)^{k-i}\right]-\left(I-P_{e}\right)^{B}\right\}
\end{aligned}
$$

If $\mathrm{N}=3$ and $\mathrm{h}=1$, then $P_{A}$ is given by

$$
\begin{aligned}
& P_{A}=\left[C_{1}^{k} P_{I}^{k-1} P_{3} C_{2}^{B-k} P_{1}^{B-k-2}\left(P_{2}+P_{3}\right)^{2}\right]\left\{\left[\left[\sum_{j=0}^{1-1} C_{i}^{k} P_{e}^{i}\left(I-P_{e}\right)^{k-i}\right]-\left(1-P_{e}\right)^{B}\right\}\right. \\
& +\left(C_{l}^{k} P_{l}^{k-1} P_{2} C_{2}^{B-k} P_{1}^{B-k-2}\left(2 P_{2} P_{3}+P_{3}^{2}\right)\right\}\left[\left[\sum_{i=0}^{1} C_{i}^{k} P_{e}^{i}\left(1-P_{e}\right)^{k-1}\right]-\left(I-P_{e}\right)^{B}\right\} \\
& \left.=\mid C_{1}^{k} C_{2}^{B-k} P_{1}^{B-3} P_{3}\left(P_{2}+P_{3}\right)^{2}\right]\left[\left[\sum_{j=0}^{1-1} C_{i}^{k} P_{e}^{i}\left(1-P_{e}\right)^{k-i}\right]-\left(1-P_{e}\right)^{B}\right\} \\
& +\left(C_{1}^{k} C_{2}^{B-k} P_{1}^{B-3} P_{2}\left(P_{2}+P_{3}\right)^{2}-P_{2}^{2}\right)\left[\left\{\left[\sum_{j=0}^{1} C_{i}^{k} P_{e}^{i}\left(1-P_{e}\right)^{k-i}\right]-\left(1-P_{e}\right)^{B}\right\}\right.
\end{aligned}
$$

If $\mathrm{N}=\mathrm{n}$ and $\mathrm{h}=1$, then $P_{A}$ can be expressed as

$$
\begin{aligned}
& P_{A}=\left[C_{l}^{k} C_{n-l}^{B-k} P_{I}^{B-1} P_{3}\left(P_{2}+P_{3}\right)^{n-1}\right)\left[\left[\sum_{j=0}^{t-1} C_{i}^{k} P_{e}^{i}\left(1-P_{e}\right)^{k-i}\right]-\left(1-P_{e}\right)^{B}\right\} \\
& +C_{1}^{k} C_{n-1}^{B-k} P_{1}^{B-3} P_{2}\left[\left(P_{2}+P_{3}\right)^{n-1}-P_{2}^{n-1}\right\}\left\{\left[\left\{\sum_{j=0}^{C} C_{i}^{k} P_{e}^{i}\left(1-P_{e}\right)^{k-i}\right]-\left(1-P_{e}\right)^{B}\right\}\right.
\end{aligned}
$$

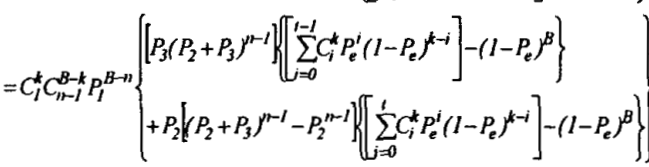

Therefore, when $\mathrm{h}=1$, the total probability of $P_{A}$ can be expressed as

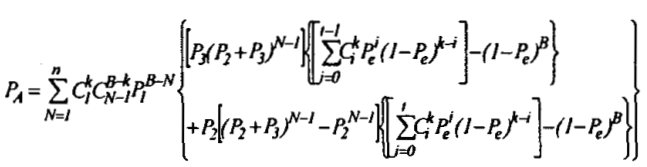

Then, when $\mathrm{h}=2$, the total probability of $P_{A}$ can be derived as 


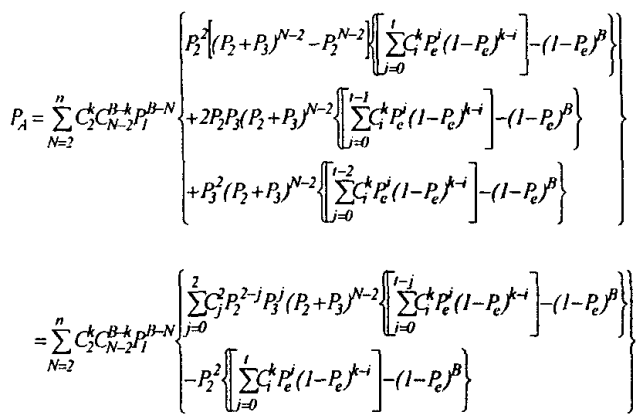

Therefore, the total probability of $P_{A}$ is given by

$$
\begin{aligned}
& p_{A}=\sum_{h=N=h}^{n} \sum_{h}^{n} C_{h}^{k} C_{N-h}^{B-h} p_{1}^{B-N} \cdot \\
&\left\{\begin{array}{l}
\sum_{j=o}^{n} C_{j}^{h} p_{2}^{h-j} p_{3}^{j}\left(p_{2}+p_{3}\right)^{N-h}\left[\sum_{i=0}^{t-1} C_{i}^{k} p_{e}^{i}\left(1-p_{e}\right)^{k-i}-\left(1-p_{e}\right)^{B}\right] \\
-p_{2}^{N}\left[\sum_{i=0}^{t} C_{i}^{k} p_{e}^{i}\left(1-p_{e}\right)^{k-i}-\left(1-p_{e}\right)^{B}\right]
\end{array}\right\} ; j \leq t
\end{aligned}
$$

\section{REFERENCE}

[1] Andrew S. Tanenbaum, Computer Networks, Prentice Hall, New Jersey, third edition, 1996.

[2] Shu Lin and Daniel J. costello, Jr., Error Control Coding : Fundamentals and Applications, Prentice Hall, United States of America, 1983.

[3] Shu Lin and Philip S. Yu, 'A hybrid ARQ scheme with parity retransmission for error control of satellite channels', IEEE Trans. Commun. Vol. Com-30, no.7, pp. 1701 1719, July 1982.

[4] Yu-Ming Wang and Shu Lin, 'A modified selective-repeat type-II hybrid ARQ system and its performance analysis', IEEE Trans. on Comm., Vol. Com-31, No. 5, pp. 593-607, May 1983.

[5] Jingshown $\mathrm{Wu}$ and Cheing-hong Lin, 'Throughput of ATM Packet over Wireless Rayliegh Channel', proceedings of IEEE ATM workshop99, Kochi Japan, May, 1999

[6] John G. Proakis, Digital Communication, Prentice Hall, Singapore, third edition, 1995. 